TRANSACTIONS OF THE

AMERICAN MATHEMATICAL SOCIETY

Volume 187, Issue 1, 1974

\title{
TOPOLOGICAL DYNAMICS AND GROUP THEORY
}

BY

\section{SHMUEL GLASNER}

\begin{abstract}
We prove, using notions and techniques of topological dynamics, that a nonamenable group contains a finitely-generated subgroup of exponential growth. We al so show that a group which belongs to a certain class, defined by means of topological dynamical properties, always contains a free subgroup on two generators.
\end{abstract}

Topological dynamics can be viewed as a theory of representations of groups as homeomorphism groups. In this paper we want to emphasize this point of view and to show that information about the structure of a group can be obtained by studying such representations. One illustration of this method is the characterization obtained in [7] of amenable groups as groups which admit no nontrivial strongly proximal flow. (See definitions below.) Furthermore this method yields results which do not explicitly involve notions from topological dynamics, for example using the above characterization of amenable groups we prove the theorem that a nonamenable group has a finitely generated subgroup of exponential growth. (This last result also follows from the main theorem in $[8$, p. 150] and Lemma 5 in [9].)

The paper is arranged as follows. In $\$ 1$ we define the necessary notions from topological dynamics. In particular strongly proximal, totally proximal and extremely proximal flows are defined. In the second section we prove, using the notion of a strongly proximal flow, that a nonamenable group contains a finitely generated subgroup of exponential growth [9]. In the third section we show that an extremely proximal flow is both strongly proximal and totally proximal. We show that a group which has a nontrivial extremely proximal flow contains a free subgroup on two generators. Finally we show by means of examples that strong proximality does not imply total proximality nor does total proximality imply strong proximality.

I wish to thank Professor H. Furstenberg for his help and advice.

1. Definitions. A flow is a pair $(T, X)$ consisting of a locally compact topological group $T$ and a compact Hausdorff space $X$, equipped with a continuous function $T \times X \rightarrow X$, denoted $(t, x) \rightarrow t x$, which satisfies the following conditions: For all $s, t \in T$ and for all $x \in X,(s t) x=s(t x)$ and for all $x \in X e x=x$,

Received by the editors January 29, 1973.

AMS (MOS) subject classifications (1970). Primary 28A65, 54H20; Secondary 43A07, $20 \mathrm{E} 05$. 
where $e$ is the identity element of $T$. If $x$ is a point of $X$ then the set. $\{t x \mid t \epsilon$ $T\}$ is the orbit of $x$, and the flow $(T, X)$ is minimal if each orbit is dense.

A flow $(T, X)$ is proximal if given two points of $X$, say $x$ and $y$, there exists a net $t_{i}$ in $T$ s.t. $\lim t_{i} x=\lim t_{i} y$. If $(T, X)$ is minimal then it is also proximal iff the diagonal is the only minimal set of the product flow $(T, X \times X)$ $(t(x, y)=(t x, t y))$.

Denote by $M(X)$ the set of all regular Borel probability measures on $X . M(X)$ with the weak * topology induced on it as a subset of $C^{*}(X)$, is a compact Hausdorff space. Here $C^{*}(X)$ is the dual space of $C(X)$, the space of real valued continuous functions on $X$. The action of $T$ on $X$ induces an action of $T$ on $M(X)$. Thus with every flow $(T, X)$ there is associated a flow $(T, M(X))$. A minimal flow $(T, X)$ is strongly proximal if the associated flow $(T, \pi(X))$ is proximal.

The flow $(T, \Re(X))$ contains the closed invariant set $X^{\prime}$ of all point masses. As a subflow of $(T, \pi(X))$ the flow $\left(T, X^{\prime}\right)$ is isomorphic with $(T, X)$ and we shall identify these flows. It is easy to see that a minimal flow $(T, X)$ is strongly proximal iff $(T, X)$ is the unique minimal set of the flow $(T, \pi(X))$ or equivalently iff for every $\mu \in \mathbb{M}(X)$ there exists a net $t_{i}$ in $T$ s.t. $\lim t_{i} \mu$ is a point mass. Since $(T, X)$ is a subflow of $(T, \Re(X))$ it follows from the definition of strongly proximal flows that a strongly proximal flow is proximal. The following theorem is proved in [7].

Theorem. A locally compact topological group $T$ is amenable iff $T$ bas no. nontrivial strongly proximal flow.

A flow $(T, X)$ is trivial if $X$ contains exactly one point. Note that our definition of a strongly proximal flow differs from the definition in [7]; there, a strongly proximal flow is not necessarily minimal.

Let $2^{X}$ be the space of all closed subsets of $X$ with the usual topology. $2^{X}$ is a compact Hausdorff space and the action of $T$ on $X$ induces an action of $T$ on $2^{X}$. Thus with every flow $(T, X)$ there is associated a flow $\left(T, 2^{X}\right)$. A minimal subset of the flow $\left(T, 2^{X}\right)$ is called a quasi-factor of the flow $(T, X)$. Every minimal flow has two obvious quasi-factors $\left(T, X_{0}\right)$ and $\left(T, X_{1}\right)$, where $X_{0}=\{X\}$ and $X_{1}=\{\{x\} \mid x \in X\} .\left(T, X_{0}\right)$ is a trivial flow while $\left(T, X_{1}\right)$ is isomorphic to $(T, X)$. Again we shall identify $X_{1}$ with $X$.

A minimal flow $(T, X)$ is totally proximal if $\left(T, X_{0}\right)$ and $\left(T, X_{1}\right)$ are the only quasi-factors of $(T, X)$, or equivalently if for every $A \in 2^{X}$ there exists a net $t_{i}$ in $T$ s.t. $\lim t_{i} A$ is either a singleton or equal to $X . A$ minimal flow $(T, X)$ is extremely proximal if for every $A \in 2^{X}$ s.t. $A \neq X$, there is a net $t_{i}$ in $T$ for which $\lim t_{i} A$ is a singleton. Clearly every extremely proximal flow is also totally proximal. 
In $\$ 3$ we shall use the notions of extensions, almost 1-1 extensions, factors and disjointness of flows. The flow $(T, X)$ is an extension of the flow $(T, Y)$ or $(T, Y)$ is a factor of $(T, X)$ if there is a continuous equivariant function from $X$ onto $Y .(T, X)$ is an almost 1-1 extension of $(T, Y)$ if there is a point in $Y$ whose inverse image in $X$ is a singleton. Finally minimal flows $(T, X)$ and $(T, Y)$ are disjoint iff the flow $(T, X \times Y)$ is minimal. (As a general reference, one can consult [3], [4] and [7].) growth.

2. A nonamenable group contains a finitely generated group of exponential

Theorem 2.l. If $T$ is a nonamenable group then $T$ contains a finitely generated subgroup of exponential growth.

Proof. By the characterization of amenable groups in $\$ 1$ we know that $T$ has a nontrivial strongly proximal flow. Let $(T, X)$ be such a flow. We need the following lemmas.

Lemma 2.2. Given $\epsilon>0, x_{0} \in X$ and a function $f$ in $C(X)$ there exists a finite subset of $T,\left\{t_{1}, \cdots, t_{k}\right\}$ s.t. for every $\nu \in M(X)$ there is an $i, 1 \leq i \leq k$, for which $\left|f\left(t_{i} \nu\right)-f\left(x_{0}\right)\right|<\epsilon$. Here $f(\mu)=\int f d \mu$ for $\mu \in \mathbb{M}(X)$.

Proof. Since $(T, X)$ is strongly proximal, for every $\mu \in \mathbb{M}(X)$ there exists $t_{\mu}$ in $T$ s.t. $\left|f\left(t_{\mu} \mu\right)-f\left(x_{0}\right)\right|<\epsilon$. By continuity $\left|f\left(t_{\mu} \nu\right)-f\left(x_{0}\right)\right|<\epsilon$ for every $\nu$ in an open neighborhood $V_{\mu}$ of $\mu$ in $\Pi(X)$. The lemma is proved by choosing a finite subcovering $\left\{V_{\mu_{1}}, \ldots V_{\mu_{k}}\right\}$ of the covering $\left\{V_{\mu} \mid \mu \in M(X)\right\}$ of the compact space $M(X)$ and putting $t_{i}=t_{\mu_{i}}$.

If $\epsilon>0$ and $\left\{x_{1} \cdots x_{n}\right\}$ is a set of (not necessarily distinct) points, we shall say that a property holds for $\epsilon$-almost all of $\left\{x_{1} \ldots x_{n}\right\}$ if it holds for all points of $\left\{x_{1} \ldots x_{n}\right\}$ with the exception of at most [nt] points, where [] stands for an integral part.

Lemma 2.3. Given $x_{0} \in X, \epsilon>0$ and $U$ an open neighborbood of $x_{0}$ in $X$, there exists a finite subset $\left\{t_{1}, \cdots, t_{k}\right\}$ of $T$ s.t. for every finite subset $\left\{x_{1}, \ldots\right.$, $\left.x_{n}\right\}$ of $X . i$ can be chosen, $1 \leq i \leq k$, for which $\epsilon$-almost all of $\left\{t_{i} x_{1}, \cdots, t_{i} x_{n}\right\}$ are in $U$.

Proof. Choose an open set $V$ s.t. $x_{0} \in V \subseteq \bar{V} \subseteq U$. Let $f$ be a continuous function on $X$ which satisfies: $0 \leq f \leq 1, f(y)=1$ for all $y \in \bar{V}$, and $f(z)=0$ for all $z \in X \backslash U$. For this $f$ and for $\epsilon$ and $x_{0}$ there exist $t_{1}, \cdots, t_{k}$ in $T$ satisfying the condition of Lemma 2.1. Now let $\left\{x_{1}, \cdots, x_{n}\right\}$ be a finite subset of $X$ and let $\mu$ be the probability measure $n^{-1} \sum_{j=1}^{n} x_{j}$, then there exists an $i, 1 \leq i \leq k$, s.t. $\left|f\left(x_{0}\right)-f\left(t_{i} \mu\right)\right|<\epsilon$, and since $0 \leq f \leq 1$ 


$$
\epsilon>\left|f\left(x_{0}\right)-f\left(t_{i} \mu\right)\right|=f\left(x_{0}\right)-f\left(t_{i} \mu\right)=1-\frac{1}{n} \sum_{j=1}^{n} f\left(t_{i} x_{j}\right) .
$$

If $a$ is the number of elements of the set $\left\{t_{i} x_{1}, \cdots, t_{i} x_{n}\right\}$ which are not in $U$ then

$$
\epsilon>1-\frac{1}{n} \sum_{j=1}^{n} f\left(t_{i} x_{j}\right) \geq 1-\frac{n-a}{n}=\frac{a}{n} .
$$

Thus $n \epsilon>a$ and $[n \epsilon] \geq a$. The lemma is proved.

To complete the proof of Theorem 2.1 remember that by our assumption $(T, X)$ is not trivial, hence there exists $x_{0} \in X$ and $t \in T$ s.t. $t x_{0} \neq x_{0}$. If we choose an open neighborhood $U$ of $x_{0}$ for which $t U \cap U=\varnothing$ and an $0<\epsilon<1 / 4$, then for these $x_{0}, U$ and $\epsilon$ there exist $t_{1}, \cdots, t_{k}$ in $T$ as in Lemma 2.3. We claim that the subgroup $S$ of $T$, generated by $\left\{t, t_{1}, \cdots, t_{k}\right\}$ is of exponential growth.

If $m$ is a positive integer denote by $\phi(m)$ the number of elements of $S$ which can be expressed as words on the alphabet $\left\{t, t_{1}, \cdots, t_{k}, t^{-1}, t_{1}^{-1}, \cdots, t_{k}^{-1}\right\}$ with length less than or equal to $m$. Let $w_{1}, \cdots, w_{\phi(m)}$, be these $\phi(m)$ elements. Choose an $i, 1 \leq i \leq k$, s.t. $\epsilon$-almost all the points of $\left\{t_{i} w_{1} x_{0}, \cdots, t_{i} w_{\phi(m)} x_{0}\right\}$ are in $U$. Then $\epsilon$-almost all the points of $\left\{t t_{i} w_{1} x_{0}, \cdots, t t_{i} w_{\phi(m)}, x_{0}\right\}$ are in $t U$.

Now if $j \neq l$ then $w_{i} \neq w_{l}$ implies $t_{i} w_{j} \neq t_{i} w_{l}$ and also $t t_{i} w_{j} \neq t t_{i} w_{l}$. If $t_{i} w_{l} x_{0} \in U$ then $t t_{i} w_{l} x_{0} \in t U$ and hence $t t_{i} w_{l} \neq t_{i} w_{l}$. Thus $\phi(m+2) \geq 2(\phi(m)-[\phi(m) \epsilon]) \geq 2 \phi(m)-2[\phi(m) \cdot 1 / 4] \geq 2 \phi(m)-1 / 2 \phi(m)=(3 / 2) \phi(m)$.

The proof is now completed by induction.

Remarks. (1) The condition in Lemma 2.3 is indeed a characterization of minimal strongly proximal flows. This characterization was suggested by Professor H. Furstenberg.

(2) Theorem 2.1 supports the following two well-known conjectures.

Conjecture A. A group is nonamenable iff it contains a free subgroup on two generators.

Conjecture B. A finitely generated group not of exponential growth is a finite extension of a nilpotent group.

Conjecture B was proved for finite extensions of solvable groups ([10], [11], [1]).

(3) Is it true that a finitely generated group is of exponential growth iff it has a nontrivial minimal proximal flow?

3. Totally proximal and extremely proximal flows. We shall say that a minimal flow $(T, X)$ is an almost extension of the flow $(T, Y)$ or that $(T, Y)$ is an almost factor of $(T, X)$ if there exists a minimal flow $\left(T, X^{*}\right)$ which is an almost 1-1 extension of $(T, X)$ and has $(T, Y)$ as a factor. 
The following theorem is proved in [7].

Theorem. Let $(T, X)$ and $(T, Y)$ be minimal flows, $X$ a metric space, then $(T, X)$ and $(T, Y)$ are disjoint iff no nontrivial quasi-factor of $(T, X)$ admits $(Y, T)$ as an almost extension.

Using this theorem we have

Theorem 3.1. Let $(T, X)$ be a totally proximal flow with $X$ metric and let $(T, Y)$ be any minimal flow. Then either $(T, X)$ is disjoint from $(T, Y)$ or $(T, X)$ is an almost factor of $(T, Y)$.

Proof. By the above theorem if $(T, X)$ and $(T, Y)$ are not disjoint then a nontrivial quasi-factor of $(T, X)$ is an almost factor of $(T, Y)$. But the only nontrivial quasi-factor of $(T, X)$ is $(T, X)$ itself and the proof is complete.

Lemma 3.2. A totally proximal flow $(T, X)$ is proximal unless $X$ contains exactly two points.

Proof. Let $x$ and $y$ be points of the flow $(T, X)$. Then $A=\{x, y\}$ is a point in $2^{X}$ and if $A \neq X$ there is a net $t_{i}$ in $T$ for which $\lim t_{i} A$ is a singleton. Thus $x$ and $y$ are proximal $\left(\lim t_{i} A\right.$ cannot be equal to $X$ unless $\left.X=A\right)$.

Theorem 2.3. An extremely proximal flow $(T, X)$ is strongly proximal unless $X$ contains exactly two points.

Proof. Since the mapping Supp of a measure to its support, from $\pi(X)$. into $2^{X}$, is lower semicontinuous it is clear that if $\operatorname{Supp}(\mu) \neq X$ then there exists a net $t_{i}$ in $T$ s.t. $\lim t_{i} \mu$ is a point mass, or in other words that cls $(T \mu)$ contains a point mass. Let $\mu$ be a measure in $\pi(X)$ with $\operatorname{Supp}(\mu)=X$. Denote $\pi=\operatorname{cls}(T \mu)$. If there exists a $\nu \in \pi$ with $\operatorname{Supp}(\nu) \neq X$ then cls $(T \nu)$ and hence also cls $(T \mu)$ contain a mass point. Otherwise, for every open nonempty set $U$ there is an $\epsilon=$ $\epsilon(U)>0$ for which $\nu(\bar{U})>\epsilon$ for all $\nu \in \pi$.

Now let $x_{0}$ be some point of $X$ and $U$ a nonempty open set in $X$ s.t. $\bar{U} \neq X$. There exists a net $t_{i}$ in $T$ for which $\lim t_{i} \bar{U}$ is the singleton $\left\{x_{0}\right\}$ and we can assume that $\lim t_{i} \mu=\nu$ exists. If $V$ is any neighborhood of $x_{0}$ then eventually

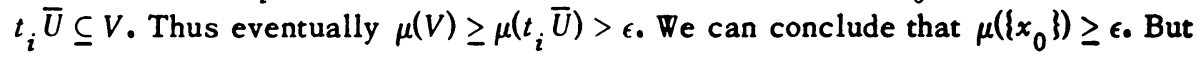
$x_{0}$ was an arbitrary point in $X$ and thus $\mu(\{x\}) \geq \epsilon$ for every point of $X$. This can be true only in the case that $X$ is finite, and by Lemma 3.2 we can conclude that $X$ must then contain exactly two points.

Theorem 3.4. If $T$ is a group which has a nontrivial extremely proximal flow $(T, X)$, then $T$ contains a free subgroup on two generators. (Nontrivial means here that $X$ contains more than two points.)(1)

(1) This was independently proved by W. A. Veech. 
Proof. Choose nonempty open sets $U, V, U_{1}, U_{2}, V_{1}$ and $V_{2}$ in $X$ s.t. $U_{1}$, $U_{2} \subseteq U, V_{1}, V_{2} \subseteq V$ and $U \cap V=U_{1} \cap U_{2}=V_{1} \cap V_{2}=\varnothing$. By extreme proximality there are $t$ and $s$ in $T$ s.t. $t\left(X \backslash U_{1}\right) \subseteq U_{2}$ and $s\left(X \backslash V_{1}\right) \subseteq V_{2}$. It is easy to see that also $t^{-1}\left(X \backslash U_{2}\right) \subseteq U_{1}$ and $s^{-1}\left(X \backslash V_{2}\right) \subseteq V_{1}$. Let $S$ be the subgroup of $T$ generated by $s$ and $t$. There is a homomorphism of $F_{2}$, the free group on two generators $a$ and $b$, onto $S$, defined by mapping $a$ on $t$ and $b$ on $s$. We claim that under this homomorphism a reduced word of the form $w=a^{i{ }^{1}} b^{j_{1}} \ldots$ cannot be mapped on an element of $S$ which acts as the identity homeomorphism on $X$. The reason for that is that since $i_{1} \neq 0, \bar{w}(X \backslash U) \subseteq U$, where $\bar{w}$ is the image of $w$ in S. Similarly a reduced word of the form $v=\bar{b}^{i_{1}} a^{j_{1}} \ldots$ cannot be mapped on an element of $S$ which acts as the identity homeomorphism on $X$ since $\bar{v}(X \backslash V) \subseteq V$. Thus the above homomorphism is an isomorphism and the theorem is proved.

We conclude with some examples. Let $X=\{\exp (2 \pi i \theta) \mid \theta$ real $\}$ and define $t \exp (2 \pi i \theta)=\exp (2 \pi i(\theta+\alpha))$ for some irrational $\alpha$. Define $s \exp (2 \pi i \theta)=$ $\exp \left(2 \pi i(\theta-[\theta])^{2}\right)$ where $[\theta]$ is the integral part of $\theta$. It is easy to see that if $T$ is the group of homeomorphisms of $X$ generated by $t$ and $s$ then the flow $(T, X)$ is extremely proximal. If $F_{2}$ is the free group on two generators $a$ and $b$, then using the homomorphism of $F_{2}$ onto $T$ defined by mapping $a$ onto $t$ and $b$ onto $s$, this flow can be considered as a flow of $F_{2}$. Let $Y$ be the compact Hausdorff space of infinite words on the symbols $a, b, a^{-1}$ and $b^{-1}$ (see, for example, [2] or [5]). Then $F_{2}$ acts naturally on $Y$ and again the resulting flow $\left(F_{2}, Y\right)$ is easily seen to be extremely proximal.

Now we shall show that the product flow $\left(F_{2}, X \times Y\right)$ is minimal. This will imply that the product flow is strongly proximal, yet it will not be a totally proximal flow since it has proper factors and hence also proper quasi-factors. So let $W$ be an invariant closed subset of $X \times Y$. If $y_{0}$ is the point aaa $\cdots$ of $Y$, then there exists $x_{0}$ in $X$ s.t. $\left(x_{0}, y_{0}\right) \in W$. Denote by $A$ the subgroup of $F_{2}$ generated by $a$, then

$$
X \times\left\{y_{0}\right\} \subseteq \operatorname{cls} A\left(x_{0}, y_{0}\right) \subseteq W .
$$

Since $\left(F_{2}, Y\right)$ is a minimal flow this implies $W=X \times Y$ and since $W$ was arbitrary $\left(F_{2}, X \times Y\right)$ is a minimal flow.

Another example of a strongly proximal flow which is not totally proximal is the homogeneous flow of $\mathrm{SL}_{3}(\mathrm{R})$ on $\mathrm{P}^{2}$, the projective space of lines through the origin in $R^{3}$. This flow is a boundary of $\mathrm{SL}_{3}(\mathbf{R})$ [6] hence strongly proximal, yet the family of closed subsets of $\mathbf{P}^{2}$ which consists of sets containing all lines belonging to some plane through the origin in $\mathbf{R}^{3}$, is a proper quasi-factor of $\left(\mathrm{SL}_{3}(\mathbf{R}), \mathbf{P}^{2}\right)$.

Since every extremely proximal flow of $\mathrm{SL}_{3}(\mathrm{R})$ is also strongly proximal and $\left(\mathrm{SL}_{3}(\mathbf{R}), \mathrm{P}^{2}\right)$ is the only nontrivial factor of the universal strongly proximal flow of 
$\mathrm{SL}_{3}(\mathrm{R})$, we can conclude that the only extremely proximal flow of $\mathrm{SL}_{3}(\mathrm{R})$ is the trivial one.

Our last example is of a flow which is totally proximal but not strongly proximal. This is a modification of the example in [7] of a flow which is minimal and proximal but not strongly proximal.

Let $X=\{0,1\}^{Z}$ and let $T$ be the group of homeomorphisms of $X$ generated by the shift on $X$ and the following set of homeomorphisms. For each basic open set

$$
B=B\left(\epsilon_{1}, \cdots, \epsilon_{n}, i_{1}, \ldots, i_{n}\right)=\left\{\xi \in X \mid \xi\left(i_{j}\right)=\epsilon_{j}, j=1, \ldots, n ; \epsilon_{j}=0 \text { or } 1\right\}
$$

and an integer $k$ s.t. $k \neq i$, for all $1 \leq j \leq n$, let $\gamma=\gamma(B, k)$ be the homeomorphism of $X$ defined by

(1) $\gamma \xi=\xi$, if $\xi \notin B$;

(2) for $\xi \in B \quad \gamma \xi(l)=\xi(l)$ if $l \neq k$ and $\gamma \xi(k)=\xi(k)^{*}$ where $1^{*}=0$ and $0^{*}=1$.

Since $T$ contains the group of homeomorphism which acts on $X$ in the example in [7], it follows that $(T, X)$ is a minimal and proximal flow. Since all the generators of $T$ preserve the Haar measure on $X,(T, X)$ is not strongly proximal. Thus all we have to show is that $(T, X)$ is totally proximal.

If $A$ is a subset of $X$ containing $2^{n}$ elements and if $\left\{B_{i}^{\prime}\right\}_{i=1}^{n}$ is the set of all possible blocks of zeroes and ones of length $n$, then we claim that there exists a $t$ in $T$ for which $t A \cap B_{i} \neq \varnothing$ for all $1 \leq i \leq 2^{n}$, where

$$
B_{i}=\left\{\xi \in X \mid \xi(0), \xi(1), \ldots, \xi(n-1)=B_{i}^{\prime}\right\} \text {. }
$$

Indeed let $A=\left\{\xi_{i}\right\}_{i=1}^{2^{n}}$, then there exists an $N>0$ such that the blocks

$$
C_{i}=\left(\xi_{i}(-N), \xi_{i}(-N+1), \ldots, \xi_{i}(0), \ldots, \xi_{i}(N)\right)
$$

are all different.

Let $\gamma_{i, j}=\gamma\left(C_{i}, N+j\right)$ if $\xi_{i}(N+j) \neq B_{i}^{\prime}(j)$, and $\gamma_{i, j}=$ identity homeomorphism of $X$, if $\xi_{i}(N+j)=B_{i}^{\prime}(j) i=1, \cdots, 2^{n} ; j=1, \cdots, 2^{n}$.

Put $t=\sigma^{N+1} \Pi_{i=1}^{2^{n}} \Pi_{j=1}^{2^{n}} \gamma_{i, j}$, where $\sigma$, defined by $(\sigma \xi)(l)=\xi(l+1)$, is the shift. Then $t A \cap B_{i} \neq \varnothing$ for $1 \leq i \leq 2^{n}$.

Now given a closed subset $A$ of $X$ we distinguish between the two cases (a) $A$ is finite, and (b) $A$ is infinite. In the first case, by the proximality of $(T, X)$ a net $t_{i}$ in $T$ exists s.t. $\lim t_{i} A$ is a singleton. In the second case by the above claim a net $t_{i}$ in $T$ can be found for which $\lim t_{i} A=X$. Thus $(T, X)$ has no proper quasi-factor, i.e. it is totally proximal.

\section{BIBLIOGRAPHY} (preprint).

1. H. Bass, The degree of polynomial growth of finitely generated nilpotent groups 
2. E. B. Dynkin and M. B. Maljutov, Random walks on groups with a finite number of generators, Dokl. Akad. Nauk SSSR 137 (1961), 1042-1045 = Soviet Math. Dokl. 2 (1961), 399-402. MR 24 \#A1751.

3. R. Ellis, Lectures on topological dynamics, Benjamin, New York, 1969. MR 42 \#2463.

4. H. Furstenberg, Disjointness in ergodic theory, minimal sets and a problem in diophantine approximation, Math. Systems Theory 1 (1967), 1-49. MR 35 \#369.

5. - Random walks and discrete sub-groups of Lie groups, Advances in Probability and Related Topics. Vol. 1, Dekker, New York, 1971, pp. 1-63. MR 44 \#1794.

6. - Poisson formula for semi-simple Lie groups, Ann. of Math. (2) 77 (1963), 335-386. MR 26 \#3820; 28 \#1246.

7. S. Glasner, Compressibility properties in topological dynamics, Amer. J. Math. (to appear).

8. H. Kesten, Full Banach mean values on countable groups, Math. Scand. 7 (1959), 146-156. MR 22 \#2911.

9. J. Milnor, $A$ note on curvature and fundamental group, J. Differential Geometry 2 (1968), 1-7. MR 38 \#636.

10. - Growth of finitely gen erated solvable groups, J. Differential Geometry 2 (1968), 447-449. MR 39 \#6212.

11. J. Wolf, Growth of finitely generated solvable groups and curvature of Riemannian manifolds, J. Differential Geometry 2 (1968), 421-446. MR 40 \# 1939.

DEPARTMENT OF MATHEMATICS, THE HEBREW UNIVERSITY OF JERUSALEM, JERUSA LEM, ISR AEL

DEPARTMENT OF MATHEMATICS, UNIVERSITY OF MINNESOTA, MINNEAPOLIS, MINNESOTA 55455 